\title{
TIPOS DE TIEMPO Y CALIDAD DEL AIRE EN TRES ESTACIONES DE FONDO DEL NW PENINSULAR
}

\author{
Samuel Sainz-Villegas, Adrián Martínez-Fernández, Ana I. Calvo, Fernanda Oduber, \\ Carlos Blanco-Alegre, Amaya Castro, Roberto Fraile \\ Departamento de Física, IMARENAB Universidad de León, 24071 León, España \\ ssainv00@estudiantes.unileon.es/amartf20@estudiantes.unileon.es/aicalg@unileon.es/fodup@unileon.es \\ cblaa@unileon.es/acasi@unileon.es/rfral@unileon.es
}

\section{Resumen}

La contaminación atmosférica se ha incrementado principalmente por el desarrollo de actividades industriales y económicas. Además, hay que tener en cuenta no sólo la contaminación atmosférica generada cerca de las fuentes, sino también la que se ha transportado hacia zonas alejadas, conocidas como regiones de fondo. En este trabajo se estudia la evolución de las concentraciones de los contaminantes atmosféricos en tres estaciones de fondo de la península ibérica y se buscan las posibles relaciones entre la concentración de contaminantes atmosféricos y las condiciones meteorológicas en dichas estaciones.

La contaminación atmosférica es un problema que afecta tanto al medio ambiente como a la salud de las personas, provocado por la emisión de partículas, sustancias o formas de energía a la atmósfera. Este problema se ha visto magnificado en gran parte por el desarrollo de actividades industriales y económicas, especialmente a lo largo del siglo pasado (Calvo, 2009). Sin embargo, según indica la Agencia Europea de Medio Ambiente (EEA), las emisiones de algunos contaminantes están sufriendo una reducción en los últimos años, como consecuencia de la aparición de nuevas políticas de contaminación atmosférica. A pesar de esto, algunas zonas urbanas, siguen registrando elevadas concentraciones de contaminantes atmosféricos (EEA, 2017).

Existe una gran diversidad de contaminantes atmosféricos entre los que podemos encontrar el ozono troposférico $\left(\mathrm{O}_{3}\right)$, los óxidos de nitrógeno $\left(\mathrm{NO}_{\mathrm{x}}\right)$, los óxidos de azufre $\left(\mathrm{SO}_{\mathrm{x}}\right)$, el monóxido de carbono $(\mathrm{CO})$, los compuestos orgánicos volátiles $(\mathrm{COV})$ y los aerosoles o material particulado (PM), entre otros (Ballester, 2005). En el presente trabajo nos centraremos especialmente en estos últimos.

Algunos estudios recientes han incluido distintos parámetros meteorológicos estacionales para explicar las fluctuaciones en las concentraciones de contaminantes en la atmósfera (Calvo et al., 2012). Variables meteorológicas tales como la velocidad y la dirección del viento o la estabilidad atmosférica influyen en la contaminación del aire. En condiciones de velocidades del viento bajas y atmósfera altamente estable, la dispersión horizontal y la mezcla vertical se ven sensiblemente reducidas y en consecuencia las concentraciones de contaminantes serán más altas (Padró-Martínez et al., 2012). Las condiciones de atmósfera estable a menudo ocurren durante el invierno (meses fríos) y a las horas previas al amanecer (Hu et al., 2009).

Algunas de las situaciones meteorológicas que pueden llegar a originar los escenarios de contaminación más dramáticos son los tipos de tiempo anticiclónicos estancados y las inversiones térmicas. 
Es importante tener en cuenta no sólo la contaminación atmosférica generada en el entorno próximo a la fuente, sino también la recibida en áreas alejadas de la misma, conocidas como regiones de fondo. Esta contaminación es consecuencia del transporte de los contaminantes por las distintas masas de aire (Poberžnik, 2016).

El principal objetivo del presente trabajo es estudiar la evolución de las concentraciones de los contaminantes atmosféricos en tres estaciones de fondo ubicadas en las localidades de O Saviñao (Lugo), Niembro (Asturias) y Peñausende (Zamora) durante los años 2001-2014 (Tabla 1). Además, se pretende establecer relaciones entre la concentración de contaminantes atmosféricos y las condiciones meteorológicas en ese mismo periodo de tiempo. Estas tres estaciones de calidad del aire pertenecen al programa EMEP (European Monitoring and Evaluation Program). Se trata de un programa europeo para el estudio de la contaminación a larga distancia, que surge a partir del Convenio sobre Contaminación Atmosférica Transfronteriza a Larga Distancia (LRTAP: LongRange Transboundary Air Pollution).

\begin{tabular}{lccccc} 
Internacional & \multicolumn{3}{c}{ la estación } & & UTM $(\mathrm{X} / \mathrm{Y})$ \\
33036999 & ES0008R & ES08 & Niembro & Asturias & $350271.5 / 4811587.41$ \\
27058999 & ES0016R & ES16 & O Saviñao & Lugo & $118345.44 / 4797213.51$ \\
49149999 & ES0013R & ES13 & Peñausende & Zamora & $259931.15 / 4574174.69$
\end{tabular}

Tabla 1.- Estaciones de fondo objeto de estudio.

En cuanto a la metodología utilizada, se procedió a la descarga de datos de las estaciones de fondo a través de la base de datos disponible en la página web del programa EMEP (www.emep.int) para posteriormente ser almacenados y tratados. En cuanto a la determinación de los tipos de tiempo, se ha utilizado una metodología basada en la clasificación de Lamb (1972). Esta se centra en los procedimientos de Jenkinson y Collison (1977) y Jones et al. (1993) utilizados para definir de una forma objetiva los diferentes tipos de tiempo en las islas británicas. La aplicación de estos métodos a la península ibérica se realiza mediante la caracterización de diferentes índices asociados a la dirección y vorticidad del flujo geostrófico de circulación diaria que afecta al oeste de la Península. Estos índices son los siguientes: flujo del sur (SF), flujo del oeste (WF), flujo total (F), vorticidad del sur (ZS), vorticidad del oeste (ZW) y vorticidad total (ZT) (Trigo y DaCamara, 2000). Estos se calcularon utilizando un total de 16 valores de presión a nivel del mar (SLP) obtenidos a partir de la serie de 16 puntos de la malla representada en la Figura 1.

Finalmente se obtiene una clasificación en 26 tipos de tiempo con 9 tipos anticiclónicos, 8 direccionales puros y 8 ciclónicos que se pueden ver en la Tabla 2 .

Los tipos de tiempo en el periodo de estudio se han caracterizado por una predominancia marcada del tipo anticiclónico (A), con un 22,65 \% del total, seguido por el tipo direccional puro NE con un 11,93\% y por el tipo direccional puro N con un 7,96\% (Fig. 2). Se ha establecido el nivel medio de contaminación para cada tipo de tiempo en cada una de las estaciones de estudio. 


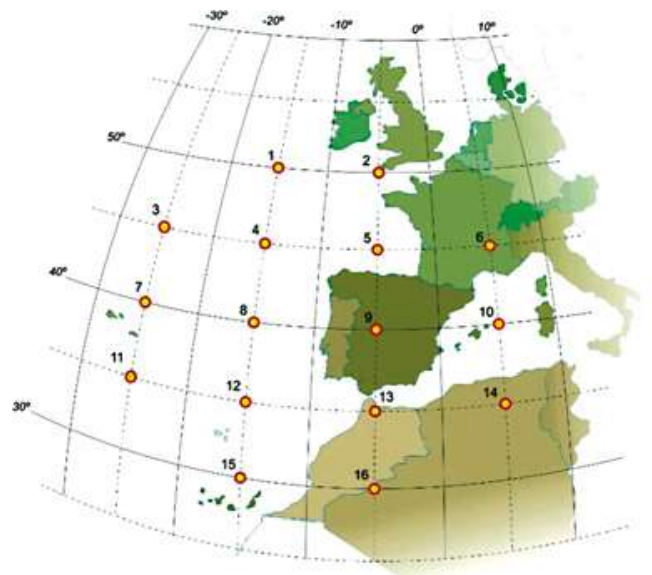

Fig. 1.- Puntos de la malla con los que se calculan los índices ZS, ZW, SF, WF (Fernández-Raga et al 2010).

\section{ANTICICLÓNICOS}

DIRECCIONALES PUROS

CICLÓNICOS

\begin{tabular}{lcccccc}
\hline A & anticiclónico & & & C & ciclónico \\
ANE & anticiclónico-noreste & NE & noreste & CNE & ciclónico-noreste \\
\hline AE & anticiclónico-este & E & este & CE & ciclónico-este \\
\hline ASE & anticiclónico-sureste & SE & sureste & CSE & ciclónico-sureste \\
AS & anticiclónico-sur & S & sur & CS & ciclónico-sur \\
ASW & anticiclónico-suroeste & SW & suroeste & CSW & ciclónico-suroeste \\
AW & anticiclónico-oeste & W & oeste & CW & ciclónico-oeste \\
ANW & anticiclónico-noroeste & NW & noroeste & CNW & ciclónico-noroeste \\
AN & anticiclónico-norte & $\mathrm{N}$ & norte & CN & ciclónico-norte
\end{tabular}

Tabla 2.- Tipos de tiempo (Fuente: Trigo y DaCamara, 2000)

La evolución de las concentraciones de los contaminantes a lo largo del periodo de estudio muestra una tendencia decreciente, como se puede apreciar en la Fig. 3, de la evolución de $\mathrm{PM}_{10}$ de las 3 estaciones. Este hecho puede ser debido a una reducción de las emisiones a la atmósfera en los núcleos urbanos, como consecuencia, principalmente, de la aplicación de la normativa tanto europea como nacional. Una reducción de la contaminación antrópica se traduce en un menor transporte de contaminantes hacia áreas alejadas de los focos de contaminación.

\section{Agradecimientos}

Este trabajo ha sido parcialmente financiado por el Ministerio de Economía y Competitividad (TEC2014-57821-R), la Universidad de León (Programa Propio 2015/00054/001) y el proyecto AERORAIN (Ministerio de Economía y Competitividad, CGL2014-52556-R, cofinanciado con fondos FEDER). Los datos fueron facilitados por el programa EMEP. F. Oduber disfruta de un contrato BES-2015-074473 del Ministerio de Economía y Competitividad. C. Blanco-Alegre disfruta de un contrato FPU16-05764 del Ministerio de Educación, Cultura y Deporte. 


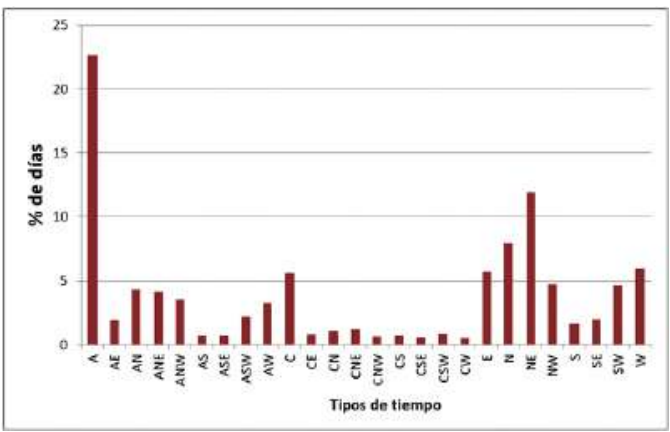

\section{Referencias}

- Ballester, F. (2005) Air Pollution, Climate Change and Health. Rev Esp Salud Pública, 79, pp. 159-175.

- Calvo I. (2009). Caracterización y transporte del aerosol atmosférica: medio urbano, rural y quema de biomasa. Tesis doctoral. Universidad de León (España).

- Calvo, A. I., Pont, V., Olmo, F. J., Castro, A., Alados-Arboledas, L., Vicente, A. M., Fernández-Raga, M.y Fraile, R. (2012) Air Masses and Weather Types: A Useful Tool for Characterizing Precipitation Chemistry and Wet Deposition. Aerosol and Air Quality Research, 12, pp. 856-878.

- EEA (2017). Contaminación atmosférica. Agencia Europea de Medio Ambiente. www.eea.europa. eu (Última visita: 09-02-2018)

- Fernández-Raga, M., Fraile, R., Keizer, JJ., Teijeiro, M.E.V., Castro, A. The kinetic energy of rain measured with an optical disdrometer: An application to splash erosion. Atmospheric Research, 96 (2-3), pp. 225-240.

- Hu, S., Fruin, S., Kozawa, K., Mara, S., Paulson, S. E. y Winer, A. M. (2009) A Wide Area of Air Pollutant Impact Downwind of a Freeway during Pre-Sunrise Hours. Atmospheric Environment, 43(16), pp. 2541-2549.

- Jenkinson, A., Collison, F. (1977) An initial climatology of gales over the North sea. Synoptic Climatology Branch Memorandum. Meteorological Office, London, 62, pp. 32.

- Jones, P., Hulme, M. and Briffa, K. (1993) A comparison of Lamb circulation types with an objective classification scheme. Int. J. Climatol., 13, pp. 655-663.

- Lamb, H. (1972) British Isles Weather types and a register of daily sequence of circulationpatterns: 1861-1971. Geophysical Memoir, HMSO London, 116, pp. 85.

- Padró-Martínez, L.T., Patton, A.P., Trull, J. B., Zamore, W., Brugge, D. y Durant, J. L. (2012) Mobile monitoring of particle number concentration and other traffic-related air pollutants in a near-highway neighborhood over the course of a year. Atmospheric Environment, 61, pp. 253-264.-

- Poberžnik, M., Strumbelj, E. (2016) The effects of air mass transport, seasonality, and meteorology on pollutant levels at the Iskrba regional background station (1996-2014). Atmospheric Environment, 134, pp. 138-146

- Trigo, R.Y. and Dacamara, C. (2000) Circulation weather types and their influence on the precipitation regime in Portugal. Int. J. Climatol., 20, pp. 1559-1581. 